\title{
Farmacologia da niacina ou ácido nicotínico
}

Pharmacology of niacin or nicotinic acid

Raul D. Santos

Unidade Clínica de Dislipidemias InCor-HCFMUSP

Niacina ou ácido nicotínico é uma vitamina solúvel com propriedades hipolipemiantes. Niacina reduz triglicérides (20\% - 50\%), LDL (5\% - 25\%), e aumenta HDL (15\% - 35\%). O estudo Coronary Drug Project mostrou que o uso de niacina foi associado com redução de eventos coronários e mortalidade total, e mais recentemente, foi demonstrado que niacina combinada com outras drogas hipolipemiantes pode atenuar a progressão da aterosclerose coronária. A niacina parece reduzir a mobilização de ácidos graxos livres dos adipócitos, agindo em receptores específicos, diminuindo a formação de lipoproteínas ricas em triglicérides pelo fígado. Existem duas formas de niacina, uma de absorção rápida (cristalina), mais comumente associada com flushing, e outra de liberação extendida, recentemente referida como de melhor tolerabilidade. 0 uso de niacina pode associar-se com dispepsia, aumento dos níveis plasmáticos de enzimas hepáticas e também com modestas elevações na glicose e ácido úrico, ao menos na utilização de doses até $2 \mathrm{~g} /$ dia da forma de liberação prolongada.

\section{Palavras-chave}

Niacina, ácido nicotínico, dislipidemia
Niacin or nicotinic acid is a soluble vitamin with hypolipidemic properties. Niacin reduces triglycerides (20 - 50\%), LDL-C (5-25\%), and raises HDL -c (15$35 \%)$. The Coronary Drug Project study showed that the use of niacin was associated with reduction on coronary events and total mortality, and more recently it has been demonstrated that niacin combined with other hypolipidemic drugs can attenuate the progression of coronary atherosclerosis.

Niacin appears to reduce the mobilization of free fatty acids from the adipocytes, acting on specific receptors, diminishing the liver formation of triglyceride-rich lipoproteins. There are two forms of niacin, one of rapid absorption (crystalline), more commonly associated with flushing, and another of extended release, recently reported to be better tolerated. The use of niacin can be associated with dyspepsia, increased plasma levels of liver enzymes and also with a modest elevation in g/ucose and uric acid plasma levels, at least using the extendedrelease preparation up to $2 \mathrm{~g} / \mathrm{d}$.

\section{KEY WORDS}

Niacin, nicotinic acid, dyslipidemia

\section{INTRODUÇÃO}

O ácido nicotínico ou niacina é uma vitamina solúvel com ação não totalmente conhecida. Existe evidência que atue sobre receptores específicos diminuindo a liberação de ácidos graxos do tecido adiposo. ${ }^{1} \mathrm{~A}$ niacina ${ }^{2}$ reduz os níveis de triglicérides (20-50\%) e de LDL colesterol (5$25 \%)$. É uma das drogas hipolipemiantes que mais aumenta o HDL colesterol (15-35\%).

Dados do Coronary Drug Project mostraram que o uso da niacina diminuiu em $27 \%$ o risco de reinfarto após cinco anos de tratamento. ${ }^{3}$ Após nove anos do final do estudo houve diminuição de $11 \%$ na mortalidade total no grupo que recebeu niacina. ${ }^{4}$ Dados dos estudos CLAS e FATS mostraram que a associação niacina colestipol reduziu a progressão angiográfica da aterosclerose. ${ }^{5,6}$ Dados do estudo HATS ${ }^{7}$ mostraram que a combinação niacina-sinvastatina foi eficaz em regredir a aterosclerose em indivíduos com HDL-C baixo. Por fim dados recentemente publicados do estudo ARBITER 2 mostram que associação niacina estatinas foi superior ao uso isolado destas últimas em diminuir a progressão da espessura intima média das carótidas. ${ }^{8}$ Considerandose que níveis baixos de HDL-C persistem como marcador de risco mesmo após o uso de estatinas, a associação estatinas-niacina pode ser uma excelente opção para o tratamento de indivíduos de risco com HDL-C persistentemente baixo. ${ }^{9}$

Atualmente existem três formulações do ácido nicotínico: liberação imediata ou cristalina, liberação intermediária, prolongada também conhecida com extendida na literatura de língua inglesa e a forma de liberação lenta. ${ }^{10}$

\section{Formas DE LIBERAÇÃo DA NIACINA}

\section{Niacina de liberação imediata}

A niacina de liberação imediata é rapidamente absorvida e excretada. Atinge pico sérico cerda de 30 a 60 minutos de sua ingestão e sua meia vida metabólica é de uma hora. Geralmente é prescrita em múltiplas doses. 


\section{Niacina de liberação lenta}

A niacina de liberação lenta, também conhecida como de liberação controlada ou sustentada, seu tempo de dissolução geralmente é maior que 12 horas.

\section{Niacina de liberação intermediária ou extendida}

Entre as duas formas clássicas de liberação da niacina encontra-se a forma de liberação intermediária ou extendida. A mesma é absorvida num período de 8 a 12 horas, intermediário entre a niacina cristalina e a lenta. A mesma deve ser ingerida uma vez por dia e é a única formulação aprovada pelo FDA para tratamento das dislipidemias. O esquema posológico para o uso desse medicamento na forma de liberação intermediária e para diminuição do "flushing" encontra-se na tabela I.

Tabela 1: Apresentação, posologia e alterações

médias \% no perfil lipídico do ácido nicotínico

\begin{tabular}{l|l|c|ccc} 
Fármaco & Apresentação & Dosagem & $\downarrow$ LDL-C & $\downarrow$ TG THDL-C \\
Niacina & Comprimidos & $1000-2000$ & $20-30$ & $20-50$ & $15-35$ \\
de liberação & $250,375^{*}, 500$, & $\mathrm{mg} / \mathrm{dia}$ & & & \\
intermediária & $750^{\star} \mathrm{e} 1000 \mathrm{mg}$ & & & & \\
ou & & & & & \\
prolongada $1,2,3,4$ & & & & &
\end{tabular}

${ }^{1}$ Existem 2 esquemas de titulação da Niacina: 1-recomenda-se iniciar com 500mg durante um mês, dobrando-se para $1 \mathrm{~g}$ por mais 1 mês, em seguida aumentar para $1500 \mathrm{mg}$ por mais 4 semanas e finalmente chegar-se a dose de $2 \mathrm{~g}$ dia. 2- iniciar com 375 aumentando-se para $500 \mathrm{mg}$ na segunda semana, para $750 \mathrm{mg}$ na terceira semana e $1 \mathrm{~g}$ na quarta semana. Após um mês recomenda-se elevar a dose para $1500 \mathrm{mg}$ e posteriormente para 2 gramas. As tomadas devem ser noturnas para minimizar o efeito do "flushing". 2- O "flushing" pode ser diminuído pela tomada de aspirina ou anti-inflamatórios não hormonais 30 minutos antes. ${ }^{3}$ Deve-se evitar o consumo de bebidas quentes e álcool antes do consumo de niacina. ${ }^{4}$ A niacina cristalina não se encontra disponível no Brasil, as doses máximas são de 3-4 g/dia e devem ser administradas em três tomadas. *- dose ainda não disponível no Brasil em Março de 2005. (Referencia 13).

\section{Vias de metabolização da niacina e toxicidade}

A niacina é metabolizada por duas vias metabólicas ${ }^{10}$ : a via na qual é conjugada com a glicina para formação do ácido nicotinúrico e a via que abrange uma série de reações de óxido-redução que formam a nicotinamida e derivados pirimidínicos.

O principal efeito colateral na niacina é o "flushing", ou seja, calor e rubor e é causado pela liberação de prostaglandinas durante a formação do ácido nicotinúrico. Já a hepatotoxicidade associa-se a metabólitos da via da nicotinamida. A niacina de liberação imediata rapidamente satura a via da nicotinamida sendo preferencialmente metabolizada pela via do ácido nicotinúrico, fato que leva a alta prevalência de "flushing" e ausência de hepatotoxicidade. Já a formulação de ação lenta raramente causa rubor devido a sua preferência pela via da nicotinamida. Por outro lado, essa formulação associa-se alto risco de hepatotoxicidade dose dependente. A formulação de liberação intermediária fica num meio de liberação imediata e raramente apresenta toxicidade hepática em doses de até 2 g/dia.

\section{Efeitos colaterais da niacina}

O uso da niacina de liberação rápida é limitado pela alta taxa de efeitos colaterais (10-50\% dos casos de interrupção do tratamento): rubor, calor, prurido, náusea, dispepsia, dor abdominal e diarréia. Em comparação com a formulação de liberação rápida a forma lenta não causa "flushing", contudo, cerca de $75 \%$ dos participantes de estudos randomizados apresentaram aumento das aminotrasnferases três vezes o limite superior do normal, sendo que muitos apresentaram sinais de insuficiência hepática. Por outro lado a niacina de liberação intermediária causa "flushing" em cerca de $50 \%$ dos casos, efeito colateral que vai diminuindo com o passar do tempo. Em estudos controlados apenas $5 \%$ dos pacientes suspenderam o tratamento devido a esse fato. O aumento das aminotrasnferases duas vezes acima do limite superior do normal ocorreu em apenas $2,6 \%$ dos casos, geralmente em associação com estatinas ou colestiramina e foi reversível com a suspensão dos medicamentos. 0 uso dessa formulação não se associa a insuficiência hepática. ${ }^{10}$

Um efeito colateral descrito com o uso da niacina de liberação imediata é a hiperglicemia. Esse fato é preocupante, pois uma grande parcela de portadores de diabetes e resistência à insulina tem níveis baixos de HDL-C sendo que a niacina seria uma ótima opção terapêutica. Contudo, há evidência de que com a formulação de liberação intermediária as alterações da glicemia são discretas e transitórias. ${ }^{11}$

Outros possíveis e raros efeitos colaterais com as diversas formulações de niacina são a hiperuricemia, gota, arritmias cardíacas, tontura, calafrios, edema, cefaléia, e ativação de úlcera péptica. ${ }^{10}$

Uma outra possibilidade é a toxicidade muscular (CPK $>10$ vezes o limite superior do normal) com o uso isolado, mas principalmente com a associação niacina-estatinas. A incidência desses casos é muito baixa e acredita-se que ocorra na ordem de 2-4/1000. ${ }^{10}$ De 601 casos avaliados pelo FDA em 2002 apenas 4 (0,7\%) ocorreram pela associação. ${ }^{12}$

\section{Uso dA NIACINA NA PRÁTICA}

Calor e rubor facial são os efeitos colaterais mais freqüentes das formas de liberação rápida, podendo ser reduzido com o uso de aspirina uma hora antes da tomada ou pelo uso da forma de liberação intermediária. As tomadas noturnas do medicamento também melhoram a tolerabilidade. ${ }^{13}$ Hiperglicemia, hiperuricemia/gota e hepatotoxicidade são menos freqüentes com a apresentação de liberação intermediária e na dosagem de até $2 \mathrm{~g} /$ dia. Doença hepática crônica e gota grave são contra-indicações absolutas. A hiperuricemia e o diabetes não contra-indicam o uso do medicamento ${ }^{10}$ sendo que as alterações da glicemia são discretas com a formulação de liberação intermediária. 


\section{REFERÊNCIAS}

1- Karpe F, Frayn KN. The nicotinic acid receptor-a new mechanism for an old drug. Lancet. 2004; 363:1892-94.

2- Santos RD, Giannini SD, Fonseca FAH et al. III Diretrizes brasileiras sobre dislipidemias e diretriz de prevenção da aterosclerose do departamento de aterosclerose da sociedade brasileira de cardiologia. Arq Bras Cardiol. 2001;77 (supl III):1-48.

3- Coronary Drug Project Research Group. Clofibrate and niacin in coronary heart disease. JAMA. 1975;231:360-381.

4- Canner PL, Berge KG, Wenger NK, Stamler J, Friedman L, Prineas RJ, Friedewald W. Fifteen year mortality in Coronary Drug Project patients: long-term benefit with niacin. J Am Coll Cardiol. 1986;8:1245-55.

5- Blankenhorn DH, Nessim SA, Johnson RL, Sanmarco ME, Azen SP, Cashin-Hemphill L. Beneficial effects of combined colestipol-niacin therapy on coronary atherosclerosis and coronary venous bypass grafts. JAMA. 1987; 257:3233-40.

6- Brown G, Albers JJ, Fisher LD et al. Regression of coronary artery disease as a result of intensive lipid-lowering therapy in men with high levels of apolipoprotein B. N Engl J Med. 1990;323:1289-98.
7- Brown BG, Zhao XQ, Chait A, et al. Simvastatin and niacin, antioxidant vitamins, or the combination for the prevention of coronary disease. N Engl J Med. 2001;345:1583-92.

8- Taylor AJ, Sullenberger LE, Lee HJ, Lee JK, Grace KA. Arterial Biology for the Investigation of the Treatment Effects of Reducing Cholesterol (ARBITER) 2: a double-blind, placebo-controlled study of extendedrelease niacin on atherosclerosis progression in secondary prevention patients treated with statins. Circulation. 2004;110:3512-7

9- Brewer HB Jr. Focus on high-density lipoproteins in reducing cardiovascular risk. Am Heart J. 2004;148(Suppl):S14-8.

10- McKenney J. New perspectives on the use of niacin in the treatment of lipid disorders. Arch Intern Med. 2004;164:697-705.

11- Grundy SM, Vega GL, McGovern E et al. Efficacy, safety, and tolerability of once-daily niacin for the treatment of dyslipidemia associated with type 2 diabetes. Arch Intern Med. 2002;162:1568-1576.

12- Shek A, Ferrill MJ. Statin-fibrate combination therapy. Ann Pharmacother. 2001;35:908-17.

13- I Diretriz Brasileira de Diagnóstico e Tratamento da Síndrome Metabólica. Hipertensão. 2004;7:126-159. 